\title{
Evolution of the neutron quasielastic scattering through the ferroelectric phase transition in $93 \% \mathrm{PbZn}_{1 / 3} \mathrm{Nb}_{2 / 3} \mathrm{O}_{3}-7 \% \mathrm{PbTiO}_{3}$
}

\author{
G.-M. Rotaru, ${ }^{1, a)}$ S. N. Gvasaliya, ${ }^{1}$ B. Roessli, ${ }^{1}$ S. Kojima, ${ }^{2}$ S. G. Lushnikov, ${ }^{3}$ and \\ P. Günter ${ }^{4}$ \\ ${ }^{1}$ Laboratory for Neutron Scattering, ETH Zurich and Paul Scherrer Institut, CH-5232 Villigen PSI, \\ Switzerland \\ ${ }^{2}$ Institute of Materials Science, University of Tsukuba, Tsukuba, Ibaraki 305-8573, Japan \\ ${ }^{3}$ Ioffe Physical Technical Institute, 26 Politekhnicheskaya, 194021 St. Petersburg, Russia \\ ${ }_{4}^{4}$ Institute for Quantum Electronics, ETH Hönggerberg-HPF, CH-8093 Zurich, Switzerland
}

(Received 11 May 2008; accepted 1 July 2008; published online 23 July 2008)

\begin{abstract}
We show that the neutron diffuse scattering in relaxor ferroelectric $(1-x) \mathrm{PZN}-x \mathrm{PT}(x=0.07)$ consists of two components. The first component is strictly elastic but extended in $q$-space and grows below $600 \mathrm{~K}$. The second component, which was not reported before for the $(1-x) \mathrm{PZN}$ $-x \mathrm{PT}$ relaxor ferroelectrics, is quasielastic with a linewidth that has a similar temperature dependence as the width of the central peak observed by Brillouin spectroscopy. The temperature dependence of the susceptibility of the quasielastic scattering has a maximum at the ferroelectric transition. () 2008 American Institute of Physics. [DOI: 10.1063/1.2963474]
\end{abstract}

Complex perovskites with the chemical formula $\mathrm{Pb} B_{1 / 3} \mathrm{Nb}_{2 / 3} \mathrm{O}_{3}(B=\mathrm{Mg}, \mathrm{Zn})$ are important materials for applications because they possess giant piezoelectric constants when doped with $\mathrm{PbTiO}_{3}$ (PT). The temperatureconcentration phase diagrams of $\mathrm{PbMg}_{1 / 3} \mathrm{Nb}_{2 / 3} \mathrm{O}_{3}$ (PMN) and $\mathrm{PbZn}_{1 / 3} \mathrm{Nb}_{2 / 3} \mathrm{O}_{3}(\mathrm{PZN})$ are complex. The average crystal structure of pure PMN is cubic at all temperatures. For small concentrations of PT there is a phase transition from cubic to rhombohedral symmetry at low temperature. Upon increasing the amount of PT a morphotropic phase boundary that separates the rhombohedral phase from a phase with tetragonal symmetry appears at $x=0.32$ (Ref. 1) for $(1-x) \mathrm{PMN}-x \mathrm{PT}$ and $x=0.08$ (Ref. 2) for $(1-x) \mathrm{PZN}-x \mathrm{PT}$. The dielectric permittivity of PMN and PZN has a broad maximum around 265 and $320 \mathrm{~K}$, respectively. At higher temperatures the dependence of the refractive index of both materials deviates from the expected linear dependence and this was explained by the appearance in the crystals of polar regions of nanometer size (PNR). ${ }^{3}$ Evidence for the formation of PNR in $(1-x) \mathrm{PMN}-x \mathrm{PT}$ and $(1-x) \mathrm{PZN}-x \mathrm{PT}$ comes from the presence of temperature-dependent diffuse scattering (DS) close to the Bragg reflections observed by both $\mathrm{x}$ rays and neutron diffraction. Upon approaching $T_{c}$, the intensity of the diffuse scattering increases which suggests that the PNRs grow and that the ferroelectric state in the relaxor ferroelectrics is eventually reached when the PNRs produce a spontaneous polarization in the same direction. Therefore it is of importance to understand the structure and dynamics of the PNRs. The local structure of the PNRs has still not be completely determined and whether or not the local symmetry of the PNRs is lower than cubic already at high temperature or undergoes a local phase transition above or at $T_{c}$ is a matter of debate. ${ }^{5,4}$ The diffuse scattering measured by neutron scattering in $(1-x) \mathrm{PMN}-x \mathrm{PT}$ is not entirely static but has an additional quasielastic (QE) component that corresponds to the dynamics of the PNRs. ${ }^{6,7}$ For PMN the intensity associated with the QE scattering was

\footnotetext{
${ }^{a)}$ Electronic mail: gelu.rotaru@psi.ch.
}

found to follow the temperature dependence of the dielectric permittivity. ${ }^{8}$ The central peak $(\mathrm{CP})$ and the QE component are also observed in doped PMN by neutron scattering, ${ }^{9}$ although the $\mathrm{CP}$ intensity is weaker than in pure PMN. In $(1-x) \mathrm{PMN}-x \mathrm{PT}$ with $x=0.32$ the susceptibility of the $\mathrm{QE}$ scattering increases below the Burns temperature and has a broad maximum at the temperature where the cubic-totetragonal phase transition occurs. ${ }^{9}$

$(1-x) \mathrm{PMN}-x \mathrm{PT}$ and $(1-x) \mathrm{PZN}-x \mathrm{PT}$ solid solutions have comparable $(x, T)$-phase diagrams and the dielectric response of both undoped materials shows relaxor properties. In PMN and in PZN, it was shown by neutron scattering that the shape and the temperature dependence of the DS are similar, and it was proposed in Refs. 8-10 that a randomfield model with cubic anisotropy could describe the physical properties of both families of compounds. In the attempt to propose a unified description of $(1-x) \mathrm{PMN}-x \mathrm{PT}$ and $(1$ $-x) \mathrm{PZN}-x \mathrm{PT}$ relaxors, an unsolved question is the behavior of the QE part of the diffuse scattering. Hlinka et al. found that the DS in $(1-x) \mathrm{PZN}-x \mathrm{PT}$ with $x=0.08$ is truly elastic, with any dynamics being slower than $8 \mathrm{GHz} .{ }^{11}$ This result contrasts with those obtained by neutron scattering from PMN and $(1-x) \mathrm{PMN}-x \mathrm{PT}(x=0.32)$ where the QE component has a lifetime varying between 0.05 and $0.2 \mathrm{THz}$ as a function of the temperature, ${ }^{6-9}$ as well as results from Brillouin experiments in $(1-x) \mathrm{PZN}-x \mathrm{PT}^{12}$

In this letter we present neutron scattering results obtained in $(1-x) \mathrm{PZN}-x \mathrm{PT}$ with $x=0.07$. We show that the low-energy part of the excitation spectrum contains in addition to the elastic DS observed by Hlinka et al. a QE component that was not reported before. We present an analysis of the evolution of the intensity and of the linewidth of this component as a function of the temperature and compare the results with those obtained in $(1-x) \mathrm{PMN}-x \mathrm{PT}(x=0$ and $x=0.32)^{8,9}$

We used the cold neutron three-axis spectrometer TASP (Ref. 14) (SINQ, ${ }^{15}$ PSI) operated in the constant $k_{f}$-mode with $k_{f}=1.64 \AA^{-1}$. A PG filter was installed in the scattered beam to reduce contaminations by higher-order wavelengths. 

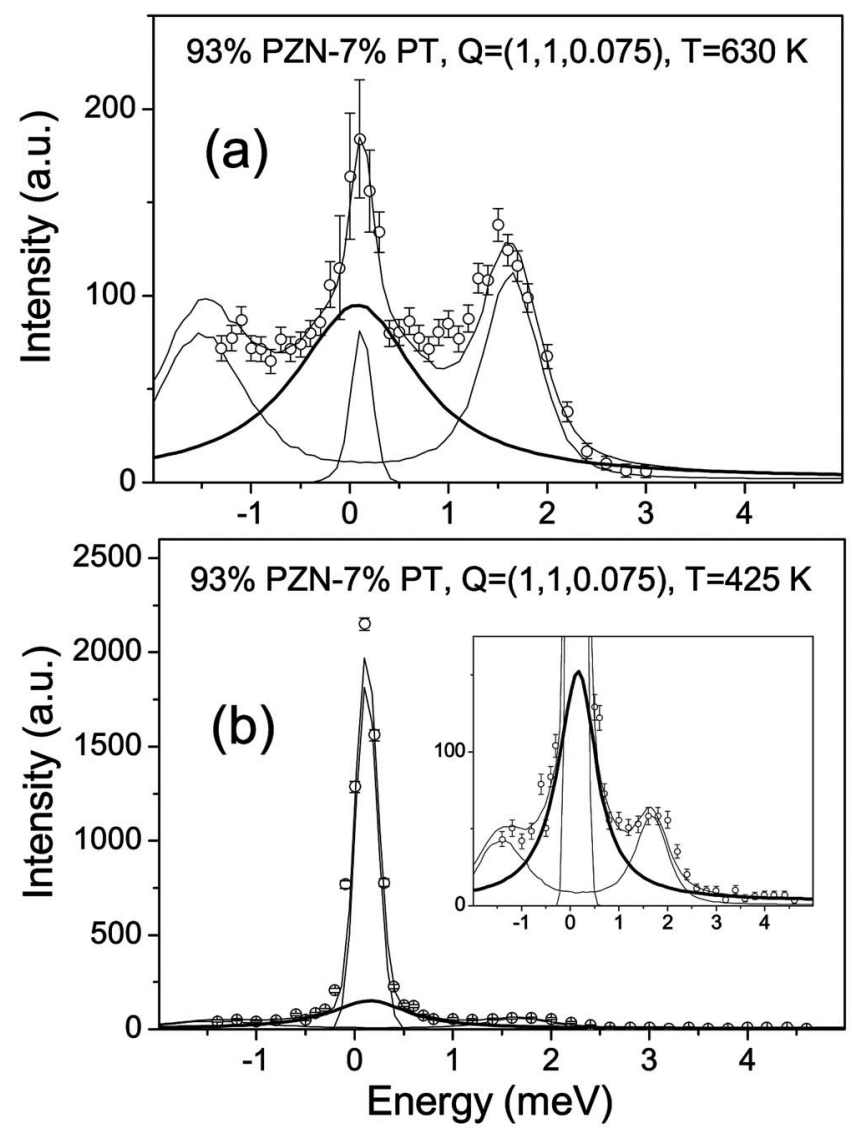

FIG. 1. Typical const $Q$ scans at $T=630 \mathrm{~K}$ (a) and at $T=425 \mathrm{~K}$ (b). The solid line is the fit. The bold line emphasizes the contribution of the $\mathrm{QE}$ component.

The horizontal collimation was $10^{\prime} / \AA-80^{\prime}-80^{\prime}-80^{\prime}$. With that setting the energy resolution was $0.26 \mathrm{meV}$. The sample $\left(\sim 1 \mathrm{~cm}^{3}\right.$ in volume) was mounted in a standard furnace with the $\langle 1 \overline{1} 0\rangle$ cubic axis vertical. Both constant- $Q$ and constantenergy scans were performed around the (1 10 ) Bragg reflection in the temperature range $300 \mathrm{~K}<T<630 \mathrm{~K}$. Figure 1(a) shows a typical energy scan performed at $T=630 \mathrm{~K}$ and $\vec{Q}=(1,1,0.075)$. The spectrum consists of a transverse acoustic (TA) phonon and of scattering centered around the elastic position. The incoherent scattering was measured at high temperature at $\vec{Q}=(1,1,0.3)$ and subtracted from the data. Figure 1(b) shows the same scan at $T=425 \mathrm{~K}$, i.e., close to the phase transition to the ferroelectric state. At this temperature the neutron intensity measured around the elastic position has strongly increased. The lineshape of the TA phonon is well described by a damped-harmonic-oscillator function convoluted with the resolution function of the spectrometer. We obtain for the TA dispersion $\omega_{\mathrm{TA}}=d \sin (\pi q)$, with $d=6.8 \pm 0.1 \mathrm{meV}$. It turns out from the analysis of the data at all temperatures that the central component cannot be reproduced by a resolution-limited Gaussian function only and consists of two components, the truly elastic diffuse scattering observed by Hlinka et al. ${ }^{11}$ and QE scattering. The QE scattering is modeled by a Lorentzian function as was done before in PMN (Refs. 6-8) and $(1-x) \mathrm{PMN}-x \mathrm{PT}^{9}$

$$
\chi_{\mathrm{QE}}^{\prime \prime}(\omega, q, T)=\frac{\chi(0, T)}{1+(q / \kappa)^{2}} \frac{\omega \Gamma_{q}}{\Gamma_{q}^{2}+\omega^{2}},
$$

where $\Gamma_{q}=\Gamma_{0}+D q^{2}$. We find that $D=18 \pm 1 \mathrm{meV}^{2}$ in $(1-x) \mathrm{PZN}-x \mathrm{PT}(x=0.07) . \kappa=1 / \xi$ is the inverse of the cor-

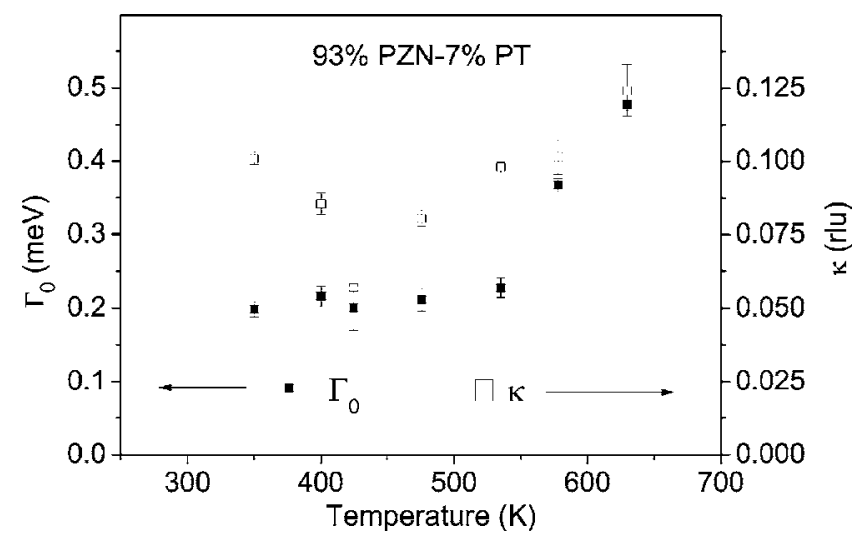

FIG. 2. The temperature dependences of the damping of the QE scattering $\Gamma_{0}$ and of the inverse correlation length $\kappa$.

relation length $\xi$ and is obtained from constant energy scans. Figure 2 shows the temperature dependence of $\kappa(T)$ and $\Gamma_{0}(T), \kappa \sim 0.12 \pm 0.01 \AA^{-1}$ at $630 \mathrm{~K}$, and decreases with decreasing temperature as does the linewidth of the QE scattering: $\Gamma_{0}=0.48 \pm 0.01 \mathrm{meV}$ at $T=630 \mathrm{~K}$ and $0.21 \pm 0.01 \mathrm{meV}$ below $\sim 475 \mathrm{~K}$, where the dynamics of the PNRs freeze. A similar behavior is observed for the temperature dependence of the width of the $\mathrm{CP}$ in light scattering where $\Gamma_{0} \sim 80 \mathrm{GHz}(\sim 0.3 \mathrm{meV})$ in the ferroelectric phase. ${ }^{12}$ On the other hand, the intensity of the elastic part of the diffuse scattering increases strongly below $600 \mathrm{~K}$ and reaches a maximum around $T_{c}$, as shown in Fig. 3. The temperature dependence of the susceptibility of the QE scattering is shown in Fig. 4. $\chi(0, T)$ increases strongly on cooling below $630 \mathrm{~K}$, reaches a maximum around $T_{c}$, and decreases rapidly in the ferroelectric phase. The temperature dependence of the susceptibility of the $\mathrm{QE}$ scattering in $(1-x) \mathrm{PZN}-x \mathrm{PT}$ $(x=0.07)$ as a similar temperature dependence as the dielectric permittivity like in $(1-x) \mathrm{PMN}-x \mathrm{PT}(x=0,0.32)$.

To conclude, we found that in $(1-x) \mathrm{PZN}-x \mathrm{PT}$ $(x=0.07)$ the DS consists of two components associated with two different timescales, which reconciles the discrepancy between light scattering ${ }^{12}$ and previous neutron scattering measurements. ${ }^{11}$ We have also shown that the temperature dependences of the $\mathrm{CP}$ and $\mathrm{QE}$ components have a similar behavior in both $(1-x) \mathrm{PMN}-x \mathrm{PT}$ and $(1-x) \mathrm{PZN}-x \mathrm{PT}$

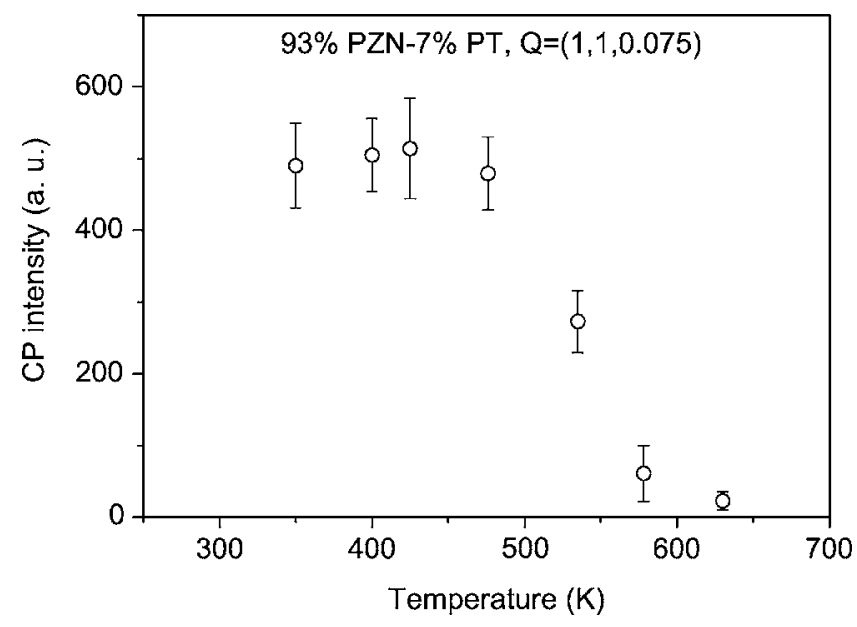

FIG. 3. The temperature dependences of the elastic component of the diffuse scattering.

o AIP license or copyright; see http://apl.aip.org/apl/copyright.jsp 


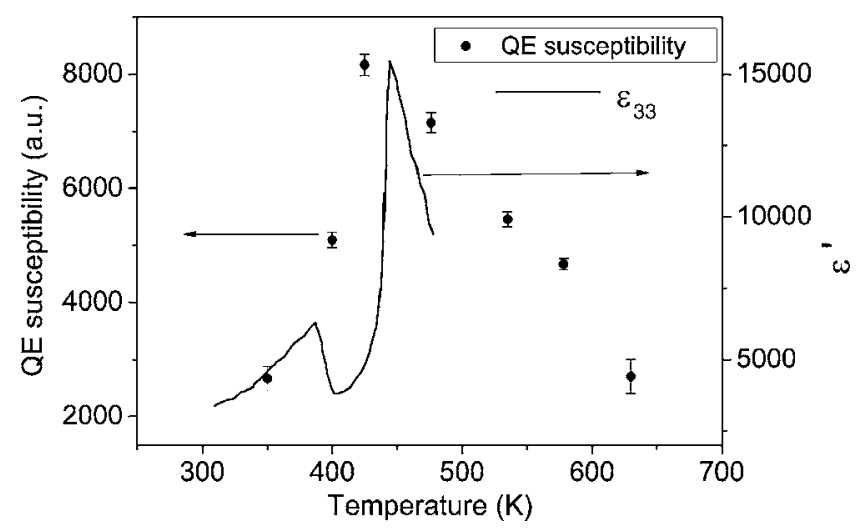

FIG. 4. The temperature dependences of the susceptibility $\chi(0, T)$ of the $\mathrm{QE}$ scattering and of the dielectric constant $\varepsilon^{\prime}$ taken from Ref. 13.

$(x=0.07)$, which brings further evidence that the static component corresponds to atomic displacements that form the PNR and the QE scattering to the dynamics of the PNR, as discussed in Refs. 6-10.

The authors thank R. A. Cowley for discussions. This work was performed at the spallation neutron source SINQ, Paul Scherrer Institut, Villigen (Switzerland) and was partially supported by the Swiss National Foundation (Project No. 20002-111545).
${ }^{1}$ V. A. Shuvaeva, A. M. Glazer, and D. Zekria, J. Phys.: Condens. Matter 17, 5709 (2005).

${ }^{2}$ J. J. Lima-Silva, I. Guedes, J. Medes Filho, A. P. Ayala, M. H. Lente, J. A. Eiras, and D. Garcia, Solid State Commun. 131, 111 (2004).

${ }^{3}$ G. Burns and F. H. Dacol, Solid State Commun. 48, 853 (1983).

${ }^{4}$ M. Roth, E. Mojaev, E. Dul'kin, P. Gemeiner, and B. Dkhil, Phys. Rev. Lett. 98, 265701 (2007).

${ }^{5}$ I.-K. Jeong, T. W. Darling, J. K. Lee, Th. Proffen, R. H. Heffner, J. S. Park, K. S. Hong, W. Dmowski, and T. Egami, Phys. Rev. Lett. 94, 147602 (2005); W. Dmowski, S. B. Vakhrushev, I.-K. Jeong, M. P. Hehlen, F. Trouw, and T. Egami, ibid. 100, 137602 (2008).

${ }^{6}$ S. N. Gvasaliya, S. G. Lushnikov, and B. Roessli, Phys. Rev. B 69, 092105 (2004).

${ }^{7}$ H. Hiraka, S.-H. Lee, P. M. Gehring, G. Xu, and G. Shirane, Phys. Rev. B 70, 184105 (2004).

${ }^{8}$ S. N. Gvasaliya, B. Roessli, R. A. Cowley, P. Huber, and S. G. Lushnikov, J. Phys.: Condens. Matter 17, 4343 (2005).

${ }^{9}$ S. N. Gvasaliya, B. Roessli, R. A. Cowley, S. Kojima, and S. G. Lushnikov, J. Phys.: Condens. Matter 19, 016219 (2007).

${ }^{10}$ C. Stock, R. J. Birgeneau, S. Wakimoto, J. S. Gardner, W. Chen, Z. G. Ye, and G. Shirane, Phys. Rev. B 69, 094104 (2004).

${ }^{11}$ J. Hlinka, S. Kamba, J. Petzelt, J. Petzelt, J. Kulda, C. A. Randall, and S. J. Zhang, J. Phys.: Condens. Matter 15, 4249 (2003).

${ }^{12}$ F. Jiang and S. Kojima, Ferroelectrics 266, 19 (2002); J.-H. Ko, D. H. Kim, and S. Kojima, Phys. Rev. B 77, 104110 (2008).

${ }^{13}$ Y. Hosono, K. Harada, T. Kobayashi, K. Itsumi, M. Izumi, Y. Yamashita, and N. Ichinose, Jpn. J. Appl. Phys., Part 1 41, 7084 (2002).

${ }^{14}$ F. Semadeni, B. Roessli, and P. Böni, Physica B 297, 152 (2001).

${ }^{15}$ W. E. Fischer, Physica B 234-236, 1202 (1997). 